\title{
Cholecystoduodenocolic Fistula by Gallbladder Carcinoma that Presenting as Bouveret's Syndrome: Case Report and Review of the Literature
}

\author{
Pasquale Cianci $^{{ }^{*}}$, Antonio Ambrosi ${ }^{1}$, Alberto Fersini ${ }^{1}$, Nicola Tartaglia ${ }^{1}$, Vincenzo Lizzi ${ }^{1}$, Francesca Sanguedolce ${ }^{2}$, \\ Alessandra Di Lascia ${ }^{1}$ and Vincenzo Neri ${ }^{1}$ \\ ${ }^{1}$ Department of Medical and Surgical Science, University of Foggia, 71122 Foggia, Italy \\ ${ }^{2}$ Department of Clinical and Experimental Medicine, University of Foggia, 71122 Foggia, Italy
}

\begin{abstract}
We describe the first case in the literature of Bouveret's syndrome as the result of a cholecystoduodenocolic fistula secondary to gallbladder carcinoma. A 67-years-old woman was admitted to our department presenting with complaints of severe cramping abdominal pain, localized predominantly in the right hypochondrium and epigastrium, with infrequent alimentary and biliary vomiting for about three months. Based on the radiological and endoscopic findings, the provisional diagnosis of Bouveret's syndrome in a patient with cholecystoduodenal fistula and history of cholelithiasis was made. The laparotomy showed the presence of the stone in the duodenal bulb, already seen at endoscopy, and adhesions between gallbladder, superior duodenal flexure and hepatic flexure of the colon, and this inflammatory gangue strethed up to the hepatic hilum. During dissection in addition to the communication between the gallbladder and the duodenum, another fistula was found that led from the gallbladder to the colon. The suspicion of malignancy of this multiple biliary enteric fistula was confirmed by intraoperative frozen sections that revealed the precence of a poorly differentiated carcinoma of biliary-pancreatic origin, but the ultimate diagnosis of gallbladder carcinoma was made exclusively after the definitive histopathological examination. For these reasons the surgical procedure involved cholecystectomy with resection of the regional lymph nodes, gastrotomy with extraction of gallstone, trans-mesocolic Roux-en-Y gastro-jejunal mechanical anastomosis, resection of the hepatic flexure of the colon with termino-lateral colo-colic mechanical anastomosis. It's important to remember that rarely the Bouveret's syndrome can hide multiple biliary enteric fistulas or even a malignancy. We believe, in the light of our experience, that the surgical approach represents the first choice in the treatment of this serious disease.
\end{abstract}

\author{
Publication History: \\ Received: June 05, 2014 \\ Accepted: July 05, 2014 \\ Published: July 07, 2014
}

\section{Keywords:}

Bouveret's syndrome,

Gastric outlet obstruction, Cholecystoduodenocolic fistula, Gallbladder carcinoma, Surgery

\section{Introduction}

Bouveret's syndrome is a rare condition characterized by gastric outlet obstruction secondary to an impacted gallstone in the duodenal bulb [1], comprising only $1-3 \%$ of cases of gallstone ileus [2], this syndrome tends to occur more commonly in women (65\%) with a median age of 74.1 years at presentation [3]. While the majority of patients with gallstones do well, approximately $6 \%$ of patients develop complications including such rare complications as fistulas [4]. The most common biliary enteric fistulas (BEFs) are cholecystoduodenal , $60 \%$ of cases; cholecystocolic, $20 \%$; and cholecystogastric, $10 \%[5]$. Bouveret's syndrome is usually the result of a cholecystoduodenal fistula. Although BEFs are not uncommon, both Bouveret's syndrome and cholecystoduodenocolic fistulas are rare and limited to case reports [6]. We describe the first case in the literature of Bouveret's syndrome as the result of a cholecystoduodenocolic fistula secondary to gallbladder carcinoma (GBC).

\section{Case Report}

A 67-years-old woman was admitted to our department presenting with complaints of severe cramping abdominal pain, localized predominantly in the right hypochondrium and epigastrium, associated with infrequent alimentary and biliary vomiting for about three months. Her vital signs were stable and there were no history of any alteration in bowel habits. The only disease was the high blood pressure treated with thiazide-like diuretic drugs. The patient was a non-smoker and non-alcoholic. Her medical history included known gallstones detected few years before on an abdominal ultrasonography performed for non-specific right upper quadrant abdominal pain.
On physical examination a vague pain was revealed in the upper quadrants of the abdomen, especially during deep palpation. Blood analysis, including liver and pancreatic function tests, were all within normal values except for an WBC of $13,52 \times 10^{\wedge} 3 / \mu \mathrm{L}$, a Lactate dehydrogenase (LDH) of $703 \mathrm{IU} / \mathrm{L}$, a Creatine phosphokinase (CPK) of $224 \mathrm{IU} / \mathrm{L}$ and a myoglobin of $135,6 \mathrm{ng} / \mathrm{ml}$. On admission coagulation tests revelead high values of fibrinogen and fibrinogen degradation products. Viral markers and tumor markers were within normal ranges. Chest and abdomen x-ray were normal except for some small air-fluid level in the right hypochondrium. The abdominal ultrasonography showed a gallbladder full of stones with thickened wall and intraluminal air and fluid pericholecystic. The fundus of the gallbladder appeared to be adherent to the superior part of the duodenum and to the superior duodenal flexure. Gastroscopy showed the presence in the duodenal bulb of a large spherical gallstone of greenish-black color. A through-the-scope balloon was passed distal to the stone and inflated but it was not possible

"Corresponding Author: Pasquale Cianci, MD, PhD, Department of Medical and Surgical Science, University of Foggia, Luigi Pinto str 1, 71122 Foggia, Italy, Tel: +390881736288 Fax: +0390881733808; E-mail: ciancidoc1@virgilio.it

Citation: Cianci P, Ambrosi A, Fersini A, Tartaglia N, Lizzi V, et al. (2014) Cholecystoduodenocolic Fistula by Gallbladder Carcinoma that Presenting as Bouveret's Syndrome: Case Report and Review of the Literature. In J Gastroenterol Disord Ther 1: 103. doi: http://dx.doi.org/10.15344/2393$849 / 2014 / 103$

Copyright: (c) 2014 Cianci et al. This is an open-access article distributed under the terms of the Creative Commons Attribution License, which permits unrestricted use, distribution, and reproduction in any medium, provided the original author and source are credited. 
Citation: Cianci P, Ambrosi A, Fersini A, Tartaglia N, Lizzi V, et al. (2014) Cholecystoduodenocolic Fistula by Gallbladder Carcinoma that Presenting as Bouveret's Syndrome: Case Report and Review of the Literature. Int J Gastroenterol Disord Ther 1: 103. doi: http://dx.doi.org/10.15344/2393-849/2014/103

to dislodge the stone and the procedure was abandoned (Figure 1). Afterwards Barium x-ray study of upper gastrointestinal tract revealed the superior duodenal flexure marked extrinsic with a thin extraluminal streak that ends with a small collection and a large stone in the first part of the duodenum (Figure 2). Abdominal computed tomography (CT), following both oral and intravenous contrast medium, was than performed. On CT, gas was seen within the gallbladder adhering to the adjacent thickened-wall first part of the duodenum with passage of oral contrast medium. The duodenal bulb contained a large gallstone of $34 \mathrm{~mm}$ which obstructed the lumen of the bowel. Rest of the abdomen was unremarkable. Some small lymph nodes of $14 \mathrm{~mm}$ were at the hepatic hilum. Based on the radiological and endoscopic findings, the provisional diagnosis of Bouveret's syndrome in a patient with cholecystoduodenal fistula and history of cholelithiasis was made. Laparotomy disclosed a mass in the right upper quadrant involving the hepatic hilum and the hepatoduodenal ligament with fistulous connections between gallbladder, superior duodenal flexure and hepatic flexure of the colon (Figure 3). Subsequently the cholecystoduodenocolic fistula was dismantled and were performed some histopathological intraoperative examinations of the gallbladder and the part of the fistula that revealed the presence of a poorly differentiated carcinoma of biliary-pancreatic origin (Figure 4). The surgery involved cholecystectomy with resection of the regional lymph nodes, omentectomy (the omentum was also incorporated into the inflammatory and neoplastic gangue),

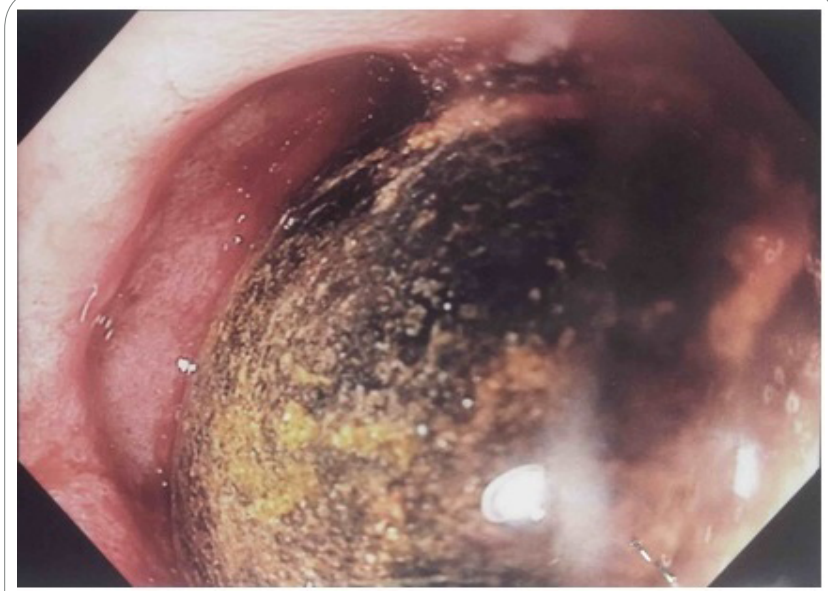

Figure 1: Gastroscopy. Presence in the duodenal bulb of a large spherical gallstone of greenish-black color.

gastrotomy with extraction of gallstone (Figure 5) and then transmesocolic Roux-en-Y gastro-jejunal mechanical anastomosis, resection of the hepatic flexure of the colon with termino-lateral colo-colic mechanical anastomosis, partial duodenoraphia with inclusion of a Foley catheter 18FR. The pathological examination of the resected specimen revealed a connectival and adipose tissue with extensive infiltration of poorly differentiated carcinoma with focal gland-like aspects. Immunohistochemically, the tumor cells were positive for CK7 and CK19, and negative for CK20, chromogranin $\mathrm{A}$ and synaptophysin. Based on the immunostains and morphology, a diagnosis of gallbladder poorly differentiated carcinoma with transmural invasion of the duodenum and colon was proferred. The patient had an uneventful postoperative course and was discharged ten days after the operation. Six weeks later, the patient returned to our department to remove the Foley catheter without complications. The multidisciplinary team with surgeons and oncologists decided to proceed with adjuvant treatment. Now the patient is in good clinical condition to begin the chemotherapy.

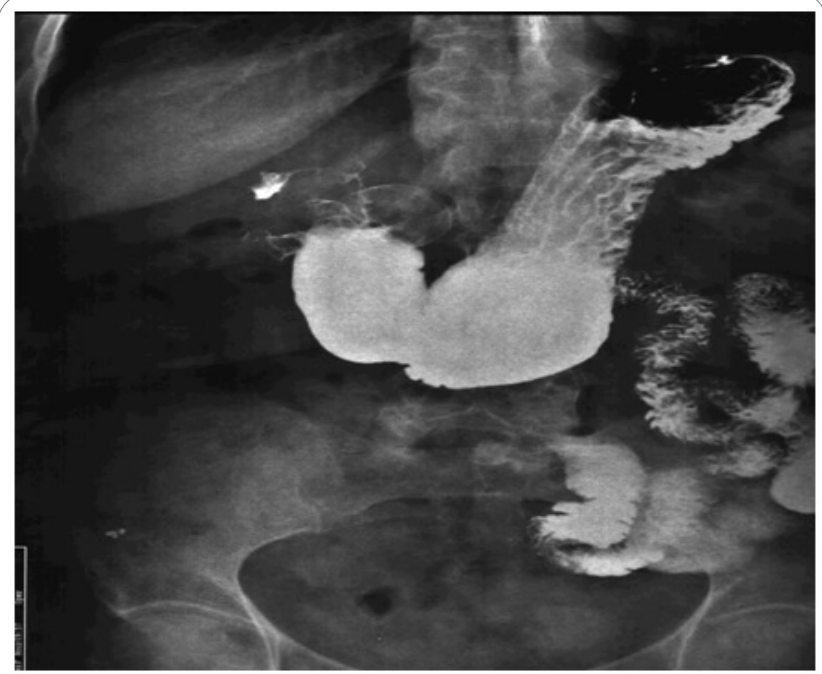

Figure 2: Barium x-ray study of upper gastrointestinal tract. The superior duodenal flexure marked extrinsic with a thin extraluminal streak that ends with a small collection and a large stone in the first part of the duodenum.

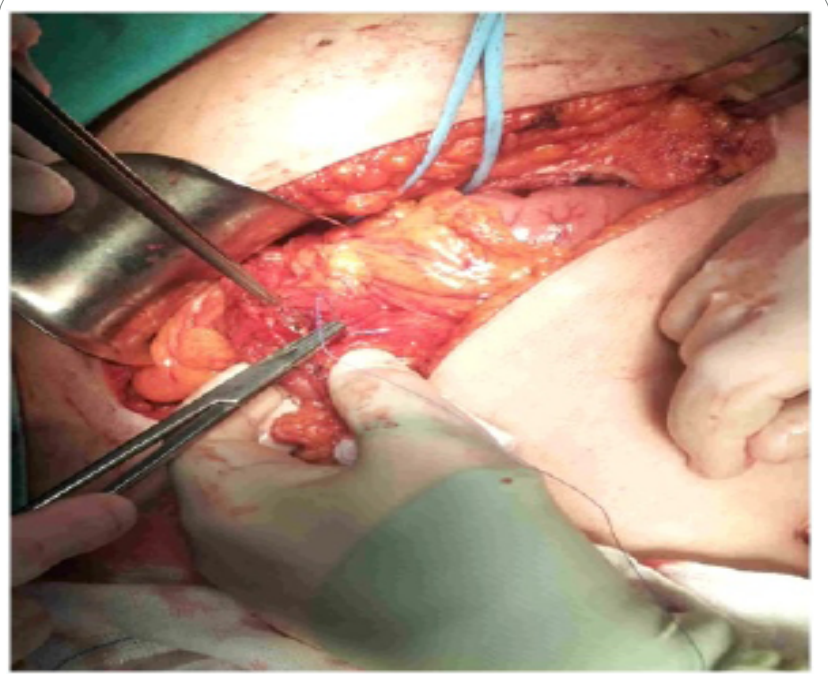

Figure 3: Intraoperative image, the cholecystocolic component of the multiple fistulas.

\section{Discussion}

In 1896, Leon Bouveret reported the first two cases of gastric outlet obstruction because of gallstones [7]. Bouveret's syndrome is an uncommon form of gallstone ileus, comprising only 1-3\% of cases [2]. The most common cause of this obstruction is a fistula, which develops after the inflamed gallbladder becomes adherent to its common anatomic resting place, the superior portion of the postbulbar duodenum [8]. Until 2008, up to 300 cases had been reported in the world literature $[9,10]$. Mortality rate, though reduced with respect to the past, has been reported to be around $12 \%$ [11]. In the past the reasons for this highest mortality were due to advanced age of patients, commonly associated comorbidities, and frequent delay in establishing the correct diagnosis due to its rarity and lack of 
Citation: Cianci P, Ambrosi A, Fersini A, Tartaglia N, Lizzi V, et al. (2014) Cholecystoduodenocolic Fistula by Gallbladder Carcinoma that Presenting as Bouveret's Syndrome: Case Report and Review of the Literature. Int J Gastroenterol Disord Ther 1: 103. doi: http://dx.doi.org/10.15344/2393-849/2014/103

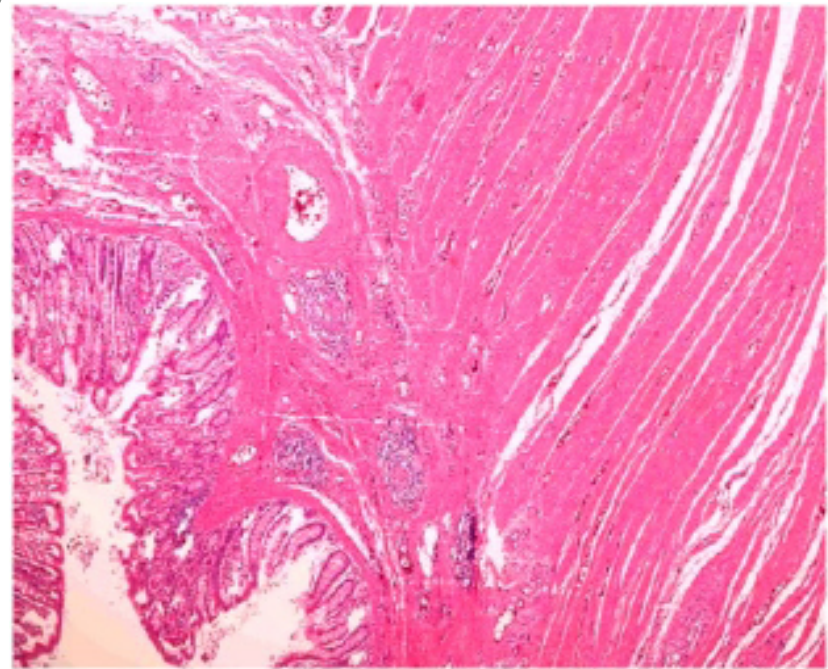

Figure 4: Histopathological intraoperative examination. Large bowel wall showing infiltrating ab extrinseco adenocarcinoma (H\&E, original magnification 40X).

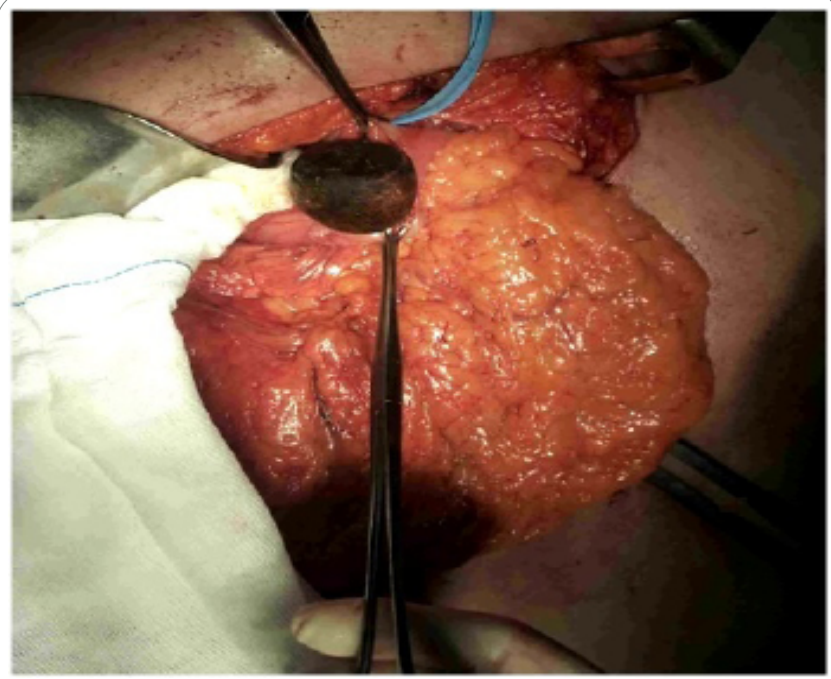

Figure 5: Intraoperative image, extraction of the gallstone by gastrotomy.

specific signs [10,12]. The overall incidence of internal biliary fistula is 1.2-5.0\% [13]. Spontaneus BEFs are a complication of biliary disease or a disease of adjacent structures. They are usually associated with gallstones; however, peptic ulcer disease, abdominal trauma, Crohn's disease, and malignancies of the biliary tract bowel and head of pancreas, have also been implicated as causes $[4,14,15]$. The most common BEFs are cholecystoduodenal, $60 \%$ of cases; cholecystocolic, $20 \%$; and cholecystogastric, $10 \%$ [5]. Bouveret's syndrome is usually the result of a cholecystoduodenal fistula, and involves female geriatric population around 65-75 years [1]. Gallstones eroding into the adjacent viscera are responsible for $75-90 \%$ of spontaneus BEF [16] The factors favoring the fistula formation are: size of the gallstone, long history of biliary disease, reported episodes of acute cholecystitis, female sex and advanced age. Cholecystoduodenocolic fistulas are extremely rare, with only 25 cases reported [5,6,16-22]. Twenty-three of these fistulas have resulted from penetrated gallstones; one was secondary to duodenal ulcer disease, and another one due to GBC. Primary GBC is responsible for less than $5 \%$ of BEF. GBC is, however, complicated by fistulization in a $3.5-4 \%$ of patients [23]. GBC is a relatively rare neoplasm which is associated with a poor prognosis, as it is often diagnosed in an advanced stage due to its nonspecific clinical presentation [24]. The gallbladder, in $80 \%$ of normal individuals, anatomically lies close to the superior medial aspect of the right colic flexure and to the second portion of the duodenum. The tumor spreads mainly by perineural, lymphatic and direct extension into adjacent viscera with the liver involved in over $60 \%$ of cases [20]. BEFs are not uncommon, conversly multiple fistulas are very rare. Morris et $\mathrm{Al}$. in 1978[20] had divided into two types the cholecystoduodenocolic fistulas. Patients who have a cholecystoduodenocolic fistula with a separate cholecystoduodenal and cholecystocolic components [25]. Secondly, multiple fistulas may exist joining more than two viscera in an abnormal connection [20]. The case that we presented belongs to the first type and regards a cholecystoduodenocolic fistula due to GBC associated with the Bouveret's syndrome. The complexity and the uniqueness of this pathological panel have made possible the diagnosis only during the surgical approach. In most cases, the presenting signs and symptoms of Bouveret's syndrome are nonspecific as: nausea, vomiting, and abdominal pain [3]. The major clinical presentation of cholecystoduodenocolic fistula is diarrhea and weight loss [20]. Even more vague, late and nonspecific are the symptoms of GBC as: abdominal pain, nausea, vomiting, jaundice and weight loss. Our patient was a woman who had abdominal pain in the right hypochondrium and epigastrium, associated with infrequent alimentary and biliary vomiting for about three months. On ultrasonography the gallbladder appeared full of stones with thickened wall and intraluminal air with pericholecystic fluid. Subsequently an endoscopic exam was performed, revealing the presence in the duodenal bulb of a large gallstone that could not be exceeded. To better assess the distal enteric tract after the stone, Barium $\mathrm{x}$-ray examination showed a fistula communicating with the superior duodenal flexure. CT scan further confirms the presence of large stone and the passage of oral contrast medium between the duodenum and the gallbladder without showing any sign of a duodenocolic fistulous component or of a malignant origin. We haven't performed a MRCP because absences of clinical signs of common bile duct involvement. Finally was made the diagnosis of Bouveret's syndrome with surgery indication. Our patient was harbouring the gallstones for a long time, and it seems reasonable that the multiple fistulas is due to inflammation of the gallbladder causing adhesions to the duodenum and to the colon with subsequent perforation into both. Erosion is probably caused by the stones. Gallstones have traditionally been regarded as an aetiological factor for GBC. Despite some epidemiological data to support this, experimental models have failed to replicate the carcinogenetic process. It is thus difficult at the present time to firmly conclude whether gallstones are the cause of GBC. Thay may however be regarded as cofactors [26]. The best therapeutic treatment of GBC is curative resection which, unfortunately, by the time of diagnosis is possible in only $15-30 \%$ of the tumors. This accounts for a 5-year survival of 7\% [23]. The optimal treatment of patients with Bouveret's syndrome still remains controversial in the world literature. The therapeutic strategy should be planned taking into consideration many parameters such as the general condition of the patient, his age and comorbidities, the location of the obstruction, the local inflammatory status, the size of the calculus and fistula[27], and the presence of more than one gallstone. The primary goal is to raise the obstruction by removing the impacted stone. This can be achieved endoscopically and surgically in laparoscopic or open approach. In well selected patients with important comorbidities and 
Citation: Cianci P, Ambrosi A, Fersini A, Tartaglia N, Lizzi V, et al. (2014) Cholecystoduodenocolic Fistula by Gallbladder Carcinoma that Presenting as Bouveret's Syndrome: Case Report and Review of the Literature. Int J Gastroenterol Disord Ther 1: 103. doi: http://dx.doi.org/10.15344/2393-849/2014/103

Page 4 of 4

major surgical risk, less aggressive methods can be attempted, such as endoscopic procedures, which however will not correct the fistula and will not prevent recurrences. More than $90 \%$ of patients require surgery [28]. With the accumulation of experience and the acquisition of adequate equipment, the laparoscopic approach, although difficult, has become possible. Sica et Al. in 2005 [10] report the first case of Bouveret's syndrome solved by laparoscopic route. Open surgery becomes indispensable after failure of repeated endoscopic attempts at stone retrieval. It remains generally the most accepted form of treatment for this condition. The surgical options include a single staged enterolithotomy or gastrotomy with concomitant cholecystectomy and repair of the fistula or an enterolithotomy alone with or without a second-stage cholecystectomy. We had performed the first option, for the treatment of this multiple fistulas, but for a T4 GBC there was no meaning for a more aggressive surgery to achieve an oncological radicality. In our case the onset of the GBC may have been subsequent or independent to cholelithiasis but, in conclusion, it is important to remember that rarely the Bouveret's syndrome can hide multiple BEFs or even a malignancy. We believe, in the light of our experience, that the surgical approach represents the first choice in the treatment of this serious disease.

\section{Author Contributions}

Pasquale Cianci and Vincenzo Lizzi wrote the paper and performed the clinical research data; Antonio Ambrosi, Alberto Fersini, Nicola Tartaglia and Vincenzo Neri performed the surgical operation; Alessandra Di Lascia made intraoperative photos and Francesca Sanguedolce made the pathological diagnosis.

\section{References}

1. Arioli D, Venturini I, Masetti M, Romagnoli E, Scarcelli A, et al. (2008) Intermittent gastric outlet obstruction due to a gallstone migrated through cholecysto-gastric fistula: a new variant of "Bouveret's syndrome". World J Gastroenterol 14: 125-128.

2. Langhorst J, Schumacher B, Deselaers T, Neuhaus H (2000) Successful endoscopic therapy of a gastric outlet obstruction due to a gallstone with intracorporeal laser lithotripsy: a case of Bouveret's syndrome. Gastrointest Endosc 51: 209-213.

3. Cappell MS, Davis M (2006) Characterization of Bouveret's syndrome: comprehensive review of 128 cases. Am J Gastroenterol 101: 2139-2146.

4. LeBlanc KA, Barr LH, Rush BM (1983) Spontaneous biliary enteric fistulas. South Med J 76: 1249-1252.

5. Doromal NM, Estacio R, Sherman H. Cholecysto-duodeno-colic fistula with gallstone ileus: report of a case. Dis Colon Rectum. 1975 18: 702-705.

6. Shocket E, Evans J, Jonas S (1970) Cholecysto-duodeno-colic fistula with gallstone ileus. Arch Surg 101: 523-526.

7. Bouveret $L$ (1896) Stenose du pylore adherent a la vesicule. Revue Medicale (Paris) 16: 1-16.

8. Liao Z, Li ZS, Ye P ((2007)) Bouveret's syndrome. Gastrointest Endosc 65: 703-704.

9. Ariche A, Czeiger D, Gortzak Y, Shaked G, Shelef I, et al. (2000) Gastric outlet obstruction by gallstone: Bouveret syndrome. Scand J Gastroenterol 35: 781-783

10. Sica GS, Sileri P, Gaspari AL (2005) Laparoscopic treatment of Bouveret's syndrome presenting as acute pancreatitis. JSLS 9:472-475.

11. Frattaroli FM, Reggio D, Guadalaxara A, Illomei G, Lomanto D, et al. (1997) Bouveret's syndrome: case report and review of the literature. Hepatogastroenterology 44: 1019-1022.
12. Gan S, Roy-Choudhury S, Agrawal S, Kumar H, Pallan A, (2008) More than meets the eye: subtle but important CT findings in Bouveret's syndrome. AJR Am J Roentgenol 191: 182-185

13. Elsas LJ, Gilat T (1965) Cholecystocolonic fistula with malabsorption. Ann Intern Med 63:481-486.

14. Chandar VP, Hookman $P$ (1980) Choledochocolonic fistula thorugh a cystic duct remnant. A case report. Am J Gastroenterol. 74: 179-181.

15. Fujitani K, Hasuike $Y$, Tsujinaka T, Mishima H, Takeda $Y$, et al. (2001) New technique of laparoscopic-assisted excision of a cholecystocolic fistula: report of a case. Surg Today 31: 740-742.

16. Nemhauser GM, Thompson JC (1966) Cholecystoduodenocolic fistula due to gallstones: case report. Ann Surg. 163: 81-85.

17. Everingham S (1927) Multiple internal biliary fistula. Surg Clin North Am 7: 1349-1350.

18. Pitman RG, Davies A (1963) The clinical and radiological features of spontaneous internal biliary fistulae. Br J Surg 50: 414-425.

19. Amoury RA, Barker HG (1966) Multiple biliary enteric fistulas. Am J Surg 111 180-185.

20. Morris SJ, Greenwald RA, Barkin JS, Tedesco FJ, Snyder R (1978) Cholecystoduodenocolic fistula secondary to carcinoma of the gallbladder. Am J Dig Dis 23: 849-852

21. Tanwar S, Mawas A, Tutton M, O'Riordan D (2008) Successful Endoscopic Management of Bouveret's Syndrome in a Patient with Cholecystoduodenocolic Fistulae. Case Rep Gastroenterol 2: 346-350.

22. Gibbons CP, Ross B (1984) Cholecystoduodenocolic fistula and gallstone ileus. Postgrad Med J 60: 698-699.

23. Vaittinen E (1970) Carcinoma of the gall-bladder. A study of 390 cases diagnosed in Finland 1953-1967. Ann Chir Gynaecol Fenn Suppl 168:1-81.

24. Dawes LG (2001) Gallbladder cancer. Cancer Treat Res. 109: 145-155.

25. Burson LC, Berliner SD (1976) Multiple simultaneous cholecystenteric fistulas. N Y State J Med 76: 955-957.

26. Shrikhande SV, Barreto SG, Singh S, Udwadia TE, Agarwal AK (2010) Cholelithiasis in gallbladder cancer: coincidence, cofactor, or cause! Eur J Surg Oncol36: 514-519.

27. lancu C, Bodea R, Al Hajjar N, Todea-lancu D, Bala O, et al. (2008) Bouveret syndrome associated with acute gangrenous cholecystitis. J Gastrointestin Liver Dis 17: 87-90.

28. Lowe AS, Stephenson S, Kay CL, May J (2005) Duodenal obstruction by gallstones (Bouveret's syndrome): a review of the literature. Endoscopy 37: 8278. 